\title{
Relationship of ovarian volume with mean platelet volume and lipid profile in patients with polycystic ovary syndrome
}

\author{
NERMIN KÖŞÜŞ, AYDIN KÖŞÜŞ and NILGÜN Ö. TURHAN \\ Department of Obstetrics and Gynecology, Faculty of Medicine, Fatih University, Ankara, Turkey
}

Received July 6, 2011; Accepted July 25, 2011

DOI: 10.3892/etm.2011.327

\begin{abstract}
The aim of the present study was to evaluate the relationship between ovarian volume $(\mathrm{OV})$ and mean platelet volume (MPV) in women with polycystic ovary syndrome (PCOS). Hormonal parameters and lipid profile of cases, and their relationship to $\mathrm{OV}$ were also assessed. Bilateral OV of 210 newly diagnosed PCOS patients and 100 healthy non-hirsute women were measured by ultrasonography. Blood samples were obtained for full blood count, hormone levels and for lipid profiles. It was found that MPV increased gradually as OV increased. This implies a higher risk of hypercoagulability and therefore an increased risk of future cardiovascular disease.
\end{abstract}

\section{Introduction}

Polycystic ovary syndrome (PCOS) is the most common female endocrinopathy, affecting $5-10 \%$ of the female population. It is an etiologically heterogeneous condition that involves overproduction of ovarian androgens leading to a heterogeneous range of symptoms, including hirsutism, acne, anovulation and infertility (1).

There are many reports pointing to causal links between PCOS and cardiovascular disease (CVD). Although the level of risk for CVD remains uncertain in PCOS, there is substantial evidence that insulin resistance, obesity, dyslipidemia, hypertension, hypercoagulable state and markers of abnormal vascular function possibly contribute to increased CVD risk (2).

Platelets are important in bleeding and coagulation disorders. The mean platelet volume (MPV), an accurate measure of platelet size, is considered a marker and determinant of platelet function. Larger platelets with higher MPV values are hemostatically more reactive and produce higher amounts of the prothrombotic factor Thromboxane A2, increasing propensity to thrombosis (3). MPV has been reported to be increased in

Correspondence to: Dr Nermin Köşüş, Ostim Mah. 1290. sok, Nevbahar Konutları A7 Blok, No. 43, Yenimahalle, Ankara, Turkey E-mail: nerminkosus@gmail.com

Key words: mean platelet volume, polycystic ovary syndrome, cardiovascular risk, ovarian volume, lipid profile patients with coronary heart disease, diabetes, atherosclerosis, hypertension and PCOS (4-8).

This study is the first to investigate the relationship between ovarian volume (OV) and MPV in women with PCOS. Hormonal parameters and lipid profile of cases, and their relationship to OV were also assessed.

\section{Patients and methods}

This prospective study was performed between January 2008 and August 2010 at the Department of Obstetrics and Gynecology, Fatih University, Faculty of Medicine. One hundred regularly menstruating healthy non-hirsute, normoovulatory women and 210 newly diagnosed PCOS patients were enrolled into the study for determination of the threshold value of OV for diagnosis of PCOS in Turkish women. All the participants were healthy women without any systemic disease. All women gave informed consent. Approval for this study was obtained from the Local Institutional Review Board of the Faculty of Medicine, Fatih University.

Rotterdam criteria were used for the diagnosis of PCOS (9). The presence of two of three of the following criteria were used for the diagnosis of PCOS: i) oligo and/or anovulation, ii) clinical and/or biochemical signs of hyperandrogenism, and iii) echographic PCO, after the exclusion of other pathologies with a similar clinical presentation. Any patient known to have hypertension, diabetes, using anti-coagulant therapy or having a propensity to thrombotic or bleeding disorders was excluded from the study group. Cases with a history of ovarian surgery, having received hormonal treatment in the previous 3 months or for PCOS-related treatment before this research were excluded from the study.

Transvaginal ultrasound examination was performed to evaluate the ovaries using a Logic 200 Pro (GE Healthcare, UK) with a $6.5-\mathrm{MHz}$ transvaginal probe. Regularly menstruating women were scanned in the early follicular phase (cycle days 3-5). Oligomenorrheic or amenorrheic women were scanned between days 3 and 5 after a progestin-induced withdrawal bleeding. Three diameters of ovaries were measured. $\mathrm{OV}$ was estimated using a simplified formula for the volume of a prolate ellipsoid: $\mathrm{V}=0.523 \mathrm{x}$ length $\mathrm{x}$ height $\mathrm{x}$ width. The mean volume of bilateral ovaries was recorded for study.

Cutoff values for OV were determined by receiver operating characteristics (ROC) curve analysis. Sensitivity against (1 - specificity) was plotted at each level, and the area under 
Table I. Demographic features, hematologic findings and mean ovarian volume of the patient groups.

\begin{tabular}{|c|c|c|c|c|c|c|}
\hline & Control & PCOS (total) & Group 1 & Group 2 & Group 3 & p-value \\
\hline Age (years) & $26.7 \pm 5.6$ & $26.3 \pm 5.4$ & $27.4 \pm 5.3$ & $25.4 \pm 7.0$ & $26.2 \pm 4.9$ & 0.117 \\
\hline BMI $\left(\mathrm{kg} / \mathrm{m}^{2}\right)$ & $20.8 \pm 2.4$ & $26.5 \pm 5.3$ & $27.1 \pm 5.5$ & $26.6 \pm 4.9$ & $25.7 \pm 5.1$ & $<0.001^{\mathrm{a}}$ \\
\hline Volume $\left(\mathrm{cm}^{3}\right)$ & $3.5 \pm 1.3$ & $8.7 \pm 1.0$ & $5.5 \pm 0.8$ & $8.2 \pm 1.0$ & $12.2 \pm 1.4$ & $<0.001^{\mathrm{a}}$ \\
\hline $\mathrm{Hb}(\mathrm{g} / \mathrm{dl})$ & $12.6 \pm 1.0$ & $12.5 \pm 1.2$ & $12.7 \pm 1.4$ & $12.4 \pm 1.1$ & $12.5 \pm 1.4$ & 0.245 \\
\hline Htc $(\%)$ & $38.1 \pm 1.7$ & $38.0 \pm 2.4$ & $37.7 \pm 2.2$ & $38.1 \pm 2.0$ & $38.2 \pm 1.9$ & 0.670 \\
\hline Plt $\left(\mathrm{x} 10^{3} / 1\right)$ & $282.1 \pm 53.4$ & $275.0 \pm 65.9$ & $292.5 \pm 58.1$ & $285.2 \pm 53.8$ & $290.1 \pm 59.7$ & 0.254 \\
\hline RDW (\%) & $13.7 \pm 2.1$ & $13.6 \pm 2.7$ & $13.3 \pm 3.0$ & $13.9 \pm 2.9$ & $13.6 \pm 2.7$ & 0.791 \\
\hline PDW (\%) & $13.6 \pm 1.0$ & $13.3 \pm 2.8$ & $12.8 \pm 3.0$ & $13.6 \pm 2.7$ & $13.1 \pm 2.6$ & 0.144 \\
\hline MPV (fL) & $8.2 \pm 1.28$ & $9.0 \pm 1.2$ & $8.46 \pm 0.83$ & $9.04 \pm 1.51$ & $9.45 \pm 0.89$ & $<0.001^{\mathrm{b}}$ \\
\hline
\end{tabular}

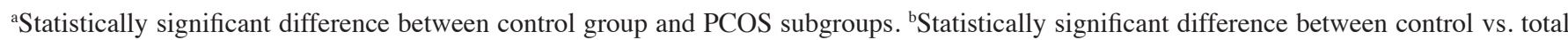
PCOS, Group 2 and Group 3; Group 1 vs. Groups 2 and 3. BMI, body-mass index; Hb, hemoglobin; Htc, hematocrit; Plt, platelet; RDW, red blood cell distribution width; PDW, platelet distribution width; MPV, mean platelet volume.

Table II. Hormonal parameters of groups (means $\pm \mathrm{SD})$.

\begin{tabular}{|c|c|c|c|c|c|}
\hline & Control & Group 1 & Group 2 & Group 3 & p-value \\
\hline DHEAS $(\mu \mathrm{g} / \mathrm{dl})$ & $223.6 \pm 45.8$ & $218.3 \pm 51.6$ & $230.8 \pm 47.8$ & $234.4 \pm 56.5$ & 0.145 \\
\hline T.Testest (ng/dl) & $33.4 \pm 7.1$ & $49.3 \pm 10.3$ & $50.9 \pm 11.2$ & $52.6 \pm 12.6$ & $<0.001^{\mathrm{a}}$ \\
\hline Prolactin $(\mathrm{ng} / \mathrm{ml})$ & $15.2 \pm 4.5$ & $14.0 \pm 5.1$ & $14.5 \pm 4.6$ & $14.7 \pm 4.4$ & 0.464 \\
\hline TSH $(\mu \mathrm{IU} / \mathrm{ml})$ & $2.2 \pm 0.6$ & $2.4 \pm 0.5$ & $2.4 \pm 0.5$ & $2.5 \pm 0.4$ & 0.284 \\
\hline LH (mIU/ml) & $5.9 \pm 1.4$ & $5.7 \pm 1.3$ & $6.2 \pm 1.5$ & $6.1 \pm 1.4$ & 0.322 \\
\hline FSH (mIU/ml) & $5.6 \pm 1.2$ & $5.9 \pm 1.4$ & $5.5 \pm 1.5$ & $6.0 \pm 1.3$ & 0.158 \\
\hline Estradiol (pg/ml) & $37.2 \pm 8.2$ & $36.7 \pm 8.5$ & $40.2 \pm 9.5$ & $39.8 \pm 8.9$ & 0.256 \\
\hline
\end{tabular}

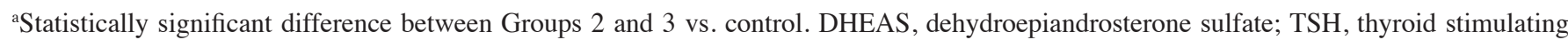
hormone; LH, luteinizing hormone; FSH, follicle stimulating hormone.

the curve (AUC), which reflects the probability of correctly identifying controls and PCOS patients, was calculated. After determination of the cutoff point for a Turkish population, patients with PCOS were divided into three subgroups according to OV. Age, weight and height of all of the cases were recorded and body mass index (BMI) was calculated.

Platelet count and MPV were performed as part of each full blood count. All samples were analyzed on a Beckman/ Coulter MAXM Hematology Analyzer (Beckman Coulter, CA, USA) 2-6 h after collection, to minimize changes in platelet size. The MPV reference range was 7.8-11.0 fL. The other parameters measured in fasting blood samples were glucose, total cholesterol, high-density lipoprotein cholesterol (HDLC), low-density lipoprotein cholesterol (LDL-C), triglyceride, FSH, LH, E2, PRL, TSH, total testosterone and DHEAS.

A power analysis based on MPV values was conducted before recruitment. Using a level of 0.05 , a power of $80 \%$ and effect size of 0.40 , a sample size of 100 individuals per group was required to detect a $10 \%$ difference between groups. All the data were analyzed on a personal computer using the SPSS 13.0 for Windows statistical package (SPSS Inc., Chicago, IL, USA). Normal distribution of the measurement values as a convenience were examined graphically using the Shapiro-Wilk test. The data are presented as proportion and the means \pm SD. The three PCOS subgroups that were formed according to OV were compared using the Kruskal-Wallis test. Significant differences that were detected in certain parameters were compared using a Bonferoni corrected Mann Whitney U test. Two-tailed $\mathrm{p}<0.05$ was considered statistically significant.

\section{Results}

The cutoff value for OV was found to be $6.43 \mathrm{~cm}^{3}$ in the Turkish population, with $95 \%$ sensitivity and $81.2 \%$ specificity. The PCOS group was divided into three subgroups according to OV. Forty-two cases having OV $<6.43 \mathrm{~cm}^{3}$ were included into Group 1, 101 cases with OV $6.43-10 \mathrm{~cm}^{3}$ were taken into Group 2 and 67 cases with larger volumes, $>10 \mathrm{~cm}^{3}$, were included into Group 3.

The mean age and BMI of the PCOS subgroups were similiar. The BMI of the control group was significantly lower than that of the other groups. Demographic features and mean $\mathrm{OV}$ of the groups are shown in Table I.

Hematologic parameters other than MPV were similiar in all subgroups and the control group (Table I). MPV values were $8.20 \pm 1.28,8.46 \pm 0.83,9.04 \pm 1.51$ and $9.45 \pm 0.89$ in the control group, Groups 1, 2 and 3, respectively. The MPV value 
Table III. Lipid profiles of the groups.

\begin{tabular}{lrrrrrr}
\hline & \multicolumn{1}{c}{ Control } & PCOS (total) & \multicolumn{1}{c}{ Group 1 } & Group 2 & Group 3 & p-value \\
\hline Total cholesterol (mg/dl) & $145.7 \pm 14.1$ & $168.1 \pm 36.5$ & $176.9 \pm 26.6$ & $153.9 \pm 30.9$ & $172.7 \pm 31.9$ & $0.005^{\text {a }}$ \\
LDL-C (mg/dl) & $75.4 \pm 13.5$ & $97.5 \pm 28.2$ & $102.3 \pm 19.8$ & $88.3 \pm 22.0$ & $99.1 \pm 28.6$ & $0.001^{\text {b }}$ \\
HDL-C (mg/dl) & $60.8 \pm 12.9$ & $51.5 \pm 12.3$ & $49.7 \pm 12.9$ & $51.5 \pm 11.0$ & $50.5 \pm 14.3$ & $0.011^{\text {b }}$ \\
Triglyceride (mg/dl) & $71.4 \pm 41.7$ & $97.9 \pm 51.9$ & $114.7 \pm 46.8$ & $83.5 \pm 32.1$ & $104.3 \pm 41.9$ & $0.009^{\text {a }}$
\end{tabular}

LDL-C, low-density lipoprotein cholesterol; HDL-C, high-density lipoprotein cholesterol. a'Statistically significant difference between control vs. whole PCOS and PCOS subgroups 1 and 3 . 'Statistically significant difference between control vs. whole PCOS and PCOS subgroups.

increased gradually as OV increased, starting from the control group up to Group 3 (Fig. 1). There were significant differences between the control group vs. Groups 2 and 3. Although the MPV of Group 1 was higher than the control group, this difference was not statistically significant (Table I). Comparison of PCOS subgroups to each other revealed significant difference in MPV between Groups 2 and 3 vs. Group $1(\mathrm{p}<0.001)$ (Table I).

Hormonal parameters and the lipid profiles of the three PCOS subgroups were similiar. When compared to the control group, there was a significant difference in terms of total testosterone level between Groups 2 and 3 vs. the control group ( $\mathrm{p}=0.004$ and $\mathrm{p}<0.001$, respectively) (Table II). Comparison of PCOS subgroups to the control group for lipid profiles revealed a significant difference in terms of LDL-C and HDL-C levels. For total cholesterol and trigylceride levels, a significant difference was observed between Groups 1 and 3 vs. the control group (Table III).

\section{Discussion}

The association between amenorrhoea and polycystic ovaries was first described by Stein and Leventhal in 1935 (10). Since then, many studies of PCOS have been conducted to better understand the ethiopathogenesis and the near/future complications of the disease. It is known that several hormonal parameters and symptoms of the disease worsen as ovarian diameter increases, which has been confirmed by studies using 3D ultrasound and conventional 2D ultrasound (11-13).

There are many reports showing the causal links between PCOS and CVD. Several studies suggest an association between PCOS and hypertension, markers of subclinical atherosclerosis and vascular dysfunction, including platelet dysfunction $(2,14-19)$.

In recent years, MPV has gained great attention due to its role in coronary heart disease, diabetes, atherosclerosis, hypertension and other vascular insufficiency states (4-8). Although direct evidence for increased thrombotic risk is lacking in PCOS patients, increased propensity to thrombosis is suggested by many studies. A propensity to thrombosis can be corrected by treatment of cases with oral contraceptives or anti-diabetic drugs (20). In the study of Kebapcilar et al (20), treatment of PCOS cases with ethinyl estradiol plus cyproterone acetate and metformin regimens resulted in a significant decrease in MPV. Only decreased insulin levels also significantly predicted the reduction of the MPV level. Thus, they speculated that decreased insulin levels may contribute to an additional improvement to a possible thrombosis risk by

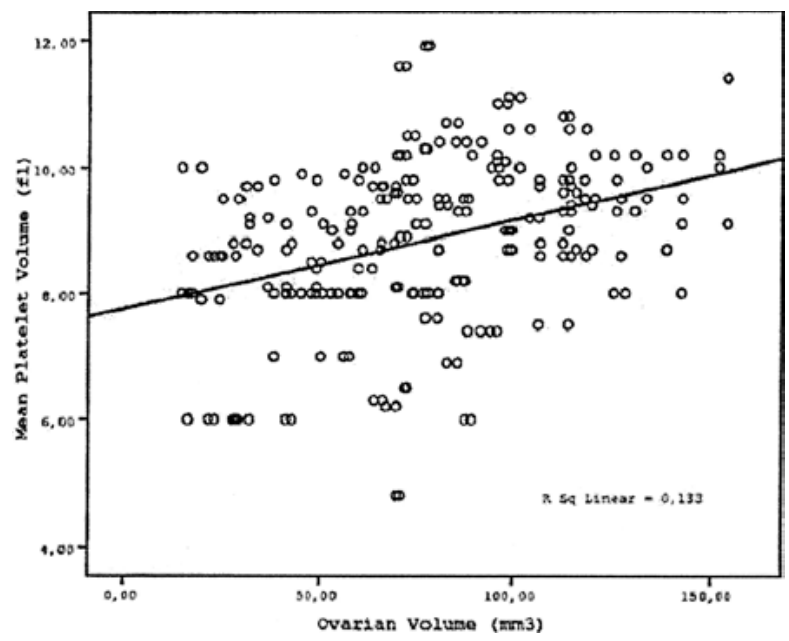

Figure 1. Relationship between ovarian volume and mean platelet volume in the PCOS cases.

reducing MPV. The improvement in hypercoagulability was observed in all patients after treatment.

Women with PCOS have abnormalities in the coagulation cascade and the fibrinolytic system (21). They have low-grade systemic coagulation and fibrinolytic activation. Several studies have demonstrated that subjects with PCOS have markedly impaired platelet responsiveness to NO, irrespective of the presence/absence of obesity. This is a completely distinct additional abnormality of platelet function to the previously described hyperaggregability, which is linked to endothelial dysfunction in such patients (22).

In a recent study of 48 patients with non-diabetic PCOS and 30 control subjects, it was shown that MPV, WBC and D-dimer levels were increased in PCOS patients, suggesting increased risk for atherosclerosis and CVD in these women (23). They also found that MPV was positively correlated with insulin levels, DHEAS and free testosterone levels in PCOS patients.

However, it remains uncertain whether PCOS-associated CVD risk is related to $\mathrm{OV}$, similar to the link between androgen levels and OV. To our knowledge, there is no study in the literature concerning the relationship of OV and MPV levels. In this study, a significant relationship was found between OV and MPV in PCOS cases. The larger the ovaries, the higher the MPV, meaning a higher risk of hypercoagulability, and so an increased risk of future CVD. We also detected that in PCOS cases with high OV, total testesterone levels also increased. The increase in MPV value may be attributed to an increase in 
androgen levels or an increase in the severity of insulin resistance as $\mathrm{OV}$ increases. A similiar relationship between $\mathrm{OV}$ and lipid profile was not detected. There may be other factors modifying lipid profiles in PCOS cases.

There are certain limitations of this study. One is the moderate sample size and the other is the absence of a comparison of different parameters related to PCOS that may effect MPV and coagulation tests. There is evidence for a pathophysiology link between MPV and insulin resistance; therefore, an important limitation of the study is the lack of measurements of serum glucose and insulin concentrations.

In conclusion, increased OV in PCOS patients may be an early sign of cardiovascular or metabolic disease later in life. Further studies are required to clarify the contribution of PCOS and increased OV to the pathogenetic process of the hematologic and coagulation system, and its direct or indirect effects on the cardiovascular system.

\section{References}

1. Homburg R: Polycystic ovary syndrome. Clin Obstet Gynaecol 2: 261-274, 2008.

2. Lo JC, Feigenbaum SL, Yang J, Pressman AR, Selby JV and Go AS: Epidemiology and adverse cardiovascular risk profile of diagnosed polycystic ovary syndrome. J Clin Endocrinol Metab 91: 1357-1363, 2006.

3. Martin JF, Trowbridge EA, Salmon G and Plumb J: The biological significance of platelet volume: its relationship to bleeding time, platelet thromboxane B2 production and megakaryocyte nuclear DNA concentration. Thrombosis Res 1: 443-460, 1983.

4. Senaran H, Ileri M, Altinbas A, Kosar A, Yetkin E, Ozturk M, Karaaslan Y and Kirazli S: Thrombopoietin and mean platelet volume in coronary artery disease. Clin Cardiol 24: 405-408, 2001.

5. Schneider DJ: Abnormalities of coagulation, platelet function, and fibrinolysis associated with syndromes of insulin resistance. Coron Artery Dis 16: 473-476, 2005.

6. Greisenegger S, Endler G, Hsieh K, Tentschert S, Mannhalter C and Lalouschek W: Is elevated mean platelet volume associated with a worse outcome in patients with acute ischemic cerebrovascular events? Stroke 35: 1688-1691, 2004

7. Endler G, Klimesch A, Sunder-Plassmann H, et al: Mean platelet volume is an independent risk factor for myocardial infarction but not for coronary artery disease. Br J Haematol 117: 399-404, 2002.

8. Gursoy A, Ertugrul DT, Pamuk B, et al: Mean platelet volume in patients with polycystic ovary disease. Platelets 17: 505-506, 2006.

9. Rotterdam ESHRE/ASRM-Sponsored PCOS Consensus Workshop Group: Revised 2003 consensus on diagnostic criteria and long-term health risks related to polycystic ovary syndrome. Fertil Steril 81: 19-25, 2004.
10. Stein IF and Leventhal ML: Amenorrhoea associated with bilateral polycystic ovaries. Am J Obst Gynecol 29: 181-191, 1935.

11. Puzigaca Z, Prelevic GM, Stretenovic Z and Balint-Peric L: Ovarian enlargement as a possible marker of androgen activity in polycystic ovary syndrome. Gynecol Endocrinol 5: 167-174, 1991.

12. Watkin KL, Tulandi T, Mathur S and LeJeune A: Threedimensional visualisation of the polycystic ovary: effect of ovarian drilling. Hum Reprod Update 2 (5), Item 18 (video), 1996.

13. Balen AH, Conway GS, Kaltsas G, Techatraisak K, Manning P, West $\mathrm{C}$ and Jacobs HS: Polycystic ovary syndrome: the spectrum of the disorder in 1741 patients. Hum Reprod 10: 2107-2111, 1995.

14. Yildiz BO, Haznedaroglu IC, Kirazli S and Bayraktar M: Global fibrinolytic capacity is decreased in polycystic ovary syndrome, suggesting a prothrombotic state. J Clin Endocrinol Metab 87: 3871-3875, 2002

15. Talbott EO, Zborowski JV, Rager JR, Boudreaux MY, Edmundowicz DA and Guzick DS: Evidence for an association between metabolic cardiovascular syndrome and coronary and aortic calcification among women with polycystic ovary syndrome. J Clin Endocrinol Metab 89: 5454-5461, 2004.

16. Kelly CJ, Speirs A, Gould GW, Petrie JR, Lyall H and Connell JM: Altered vascular function in young women with polycystic ovary syndrome. J Clin Endocrinol Metab 87: 742-746, 2002.

17. Palmert MR, Gordon CM, Kartashov AI, Legro RS, Emans SJ and Dunaif A: Screening for abnormal glucose tolerance in adolescents with polycystic ovary syndrome. J Clin Endocrinol Metab 87: 1017-1023, 2002.

18. Apridonidze T, Essah PA, Iuorno MJ and Nestler JE: Prevalence and characteristics of the metabolic syndrome in women with polycystic ovary syndrome. J Clin Endocrinol Metab 90: 1929-1935, 2005.

19. Legro RS, Urbanek M, Kunselman AR, Leiby BE and Dunaif A: Self-selected women with polycystic ovary syndrome are reproductively and metabolically abnormal and undertreated. Fertil Steril 78: 51-57, 2002.

20. Kebapcilar L, Taner CE, Kebapcilar AG, Alacacioglu A and Sari I: Comparison of four different treatment regimens on coagulation parameters, hormonal and metabolic changes in women with polycystic ovary syndrome. Arch Gynecol Obstet 281: 35-42, 2010.

21. Thompson CB, Jakubowski JA, Quinn PG, Deykin D and Valeri CR: Platelet size and age determine platelet function independently. Blood 63: 1372-1375, 1984.

22. Rajendran S, Willoughby S, Chan W, et al: Polycystic ovary syndrome is associated with severe platelet and endothelial dysfunction in both obese and lean subjects. Atherosclerosis 204: 509-514, 2009.

23. Kebapcilar L, Taner CE, Kebapcilar AG and Sari I: High mean platelet volume, low-grade systemic coagulation and fibrinolytic activation are associated with androgen and insulin levels in polycystic ovary syndrome. Arch Gynecol Obstet 280: 187-193, 2009. 